OPEN ACCESS

Edited by:

Letizia Leocani,

San Raffaele Hospital (IRCCS), Italy

Reviewed by:

Moussa Antoine Chalah,

Hôpitaux Universitaires Henri

Mondor, France

Nils Muhlert,

The University of Manchester,

United Kingdom

*Correspondence:

Ying Chen

zsxkscy@sina.com

†These authors have contributed equally to this work

Specialty section: This article was submitted to

Multiple Sclerosis and

Neuroimmunology,

a section of the journal

Frontiers in Neurology

Received: 19 November 2019

Accepted: 30 April 2021

Published: 29 July 2021

Citation:

Chen Y, Li R, Wu A, Qiu W, Hu X,

Hu Z, Yang Q and Zhou Z (2021)

Comparison of Thalamus and Basal

Ganglia Signs Between Multiple

Sclerosis and Primary Angiitis of the

Central Nervous System: An

Exploratory Study.

Front. Neurol. 12:513253.

doi: 10.3389/fneur.2021.513253

\section{Comparison of Thalamus and Basal Ganglia Signs Between Multiple Sclerosis and Primary Angiitis of the Central Nervous System: An Exploratory Study}

\author{
Ying Chen ${ }^{1 * \dagger}$, Rui $\mathrm{Li}^{2 \dagger}$, Aimin $\mathrm{Wu}^{2}$, Wei Qiu ${ }^{2}$, Xueqiang $\mathrm{Hu}^{2}$, Zhaoqi Hu${ }^{3}$, Qian Yang ${ }^{1}$ and \\ Zhiming Zhou ${ }^{1}$ \\ ${ }^{1}$ Department of Neurology, Yijishan Hospital, The First Affiliated Hospital of Wannan Medical College, Wuhu, China, \\ ${ }^{2}$ Department of Neurology, The Third Affiliated Hospital of Sun Yat-Sen University, Guangzhou, China, ${ }^{3}$ Department of \\ Orthopaedics, Wuhu Traditional Chinese Medicine Hospital, Wuhu, China
}

Based on the symptoms, especially those affecting small vessels, it is difficult to distinguish multiple sclerosis (MS) from primary angiitis of the central nervous system (PACNS). Magnetic resonance imaging (MRI) helps understand the characteristics of deep gray matter lesions (DGML) in MS and PACNS. We aimed to compare the MRI characteristics of thalamus and basal ganglia lesions between relapsing-remitting MS and PACNS. In our study, 49 relapsing-remitting MS patients and 16 PACNS with MRI-confirmed thalamus or basal ganglia lesions were enrolled. Among the DGMLs in basal ganglia, putamen had significantly higher $(P=0.037)$ involvement in PACNS than in MS. More importantly, larger lesion sizes in thalamus helps to distinguish PACNS (12.4 $\pm 4.3 \mathrm{~mm})$ from $\mathrm{MS}(7.9 \pm 3.7 \mathrm{~mm})(P=0.006)$. But using lesions in basal ganglia, researchers were unable to differentiate the two disorders. Presently, our study shows that MRI performances of deep gray matter differ between MS and PACNS.

Keywords: multiple sclerosis, primary angiitis of the central nervous system, basal ganglia, MRI, comparison

\section{INTRODUCTION}

Multiple sclerosis (MS) is a chronic inflammatory demyelinating disease of the central nervous system (CNS), which is characterized by recurrent neurological deficits, attributed to lesions scattered in the brain and spinal cord (1). The involvement of deep gray matter (GM) structures in MS is of particular interest, because the thalamus, limbic, and striatal structures are involved in all the major functional circuits in the brain and provide points of convergence across multiple cortical, limbic, brain stem, and cerebellar systems (2). Lesions in these regions are important components of MS pathology. Gray matter demyelination has been documented in the hippocampus, basal ganglia, and thalamus, and can be observed by magnetic resonance imaging (MRI) $(3,4)$. Deep gray matter abnormality measures on MRI scans have been correlated with disability and cognitive impairment $(5,6)$. 


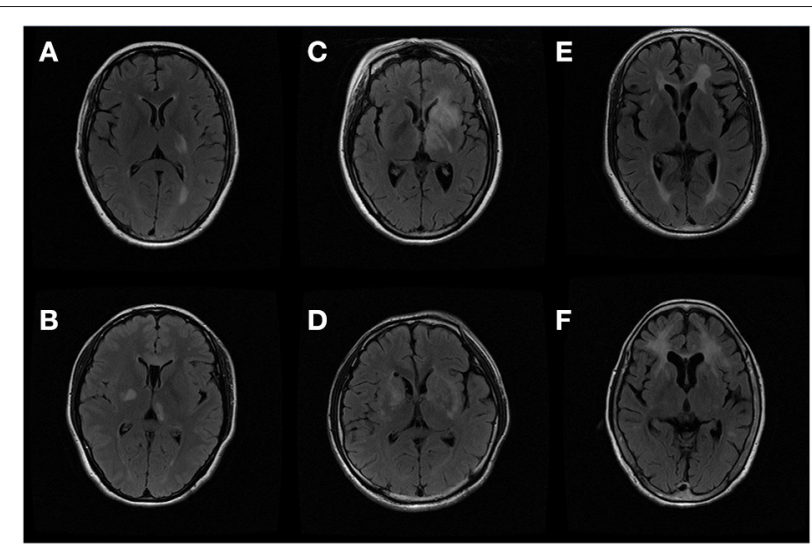

FIGURE 1 | Thalamus and basal ganglia lesions on magnetic resonance image (MRI): (A,B) thalamus lesions of multiple sclerosis (MS) and primary central nervous system angiitis (PACNS); (C,D) internal capsule lesions of MS and PACNS; (E,F) putamen lesions of MS and PACNS.

Primary angiitis of the central nervous system (PACNS) is a rare idiopathic disorder, which causes inflammation in small and medium-sized vessels (7). Patients with PACNS sometimes exhibit "MS-like" relapsing-remitting or progressive symptoms when they are affected in small vessels in acute stage $(8,9)$. Deep gray matter can also be impaired in PACNS. A study in 2016 indicated $74 \%$ of PACNS sufferers exhibited lesions in deep gray matter (10). Although these lesions could be visualized by MRI, they tend to provide the topographic MRI evidence to make a diagnosis of MS, leading to misdiagnosis (9). In this situation, the lack of a clear differentiation between MS and PACNS can be problematic.

We then made a deep investigation of MRI characteristics of basal ganglia and thalamus lesions in MS and PACNS, and compared them in this study (Figure 1).

\section{METHODS}

\section{Ethics Statement}

This study was approved by the local Ethics Committee of the Third Affiliated Hospital of Sun Yat-sen University (NO 2011-248). Informed consent for this investigation was obtained from the patients to evaluate the examination scores and standard laboratory test results that benefited the diagnosis and therapy.

\section{Patients}

We retrospectively reviewed 49 patients diagnosed with MS and 16 patients with PACNS from November 2013 to October 2018 in the MS center of the Third Affiliated Hospital of Sun Yat-sen University and the First Affiliated Hospital of Wannan Medical University. All of the patients included were adults. PACNS was diagnosed in accordance with Calabrese and Mallek in 1988 (11), and required: the presence of an acquired otherwise unexplained neurological or psychiatric deficit, the presence of either classic angiographic or histopathological features of angiitis within the CNS, and no evidence of systemic vasculitis or any disorder that could cause or mimic the angiographic or pathological features of the disease. Among them, only two patients underwent brain biopsy while the rest chose less invasive angiography. They did not meet the 2010 McDonald criteria for MS (12). The patients diagnosed with MS were from the subgroup of relapsing-remitting MS according to the 2010 McDonald criteria. Our research enrolled 49 relapsingremitting MS patients and 16 PACNS with MRI-confirmed thalamus or basal ganglia lesions. All of the patients were followed up for at least 12 months. None of the patients had systemic or inflammatory diseases elsewhere besides the central nervous system. All of the patients were given such diagnoses initially and had not received any corticosteroid before their enrollment. The decision of whether to take immunomodulatory drugs was carefully made after a thorough examination of the patients.

\section{MRI Acquisition Protocol}

A 1.5-T magnetic resonance imager (General Electric, Milwaukee, WI, USA) was used to perform MRI of the brain and spinal cord. Conventional MRI protocols were applied to all the patients: T1-weighted images (T1W) with and without gadolinium enhancement (GDE), T2weighted images (T2W), and fluid attenuated inversion recovery (FLAIR). The diameters of lesions with T2 contrast enhancement, black holes, and confluency were measured on axial sections with T2-FLAIR sequences. Patients were excluded if contraindicated for MRI or intravenous injection of gadolinium-based contrast material. MR angiography was also applied to all patients. All image archives were reviewed with a DICOM viewer on a Macintosh computer. An experienced neurologist and neuroradiologist, who were both blinded to the grouping of the two diseases and patients' clinical features, analyzed all MRI images. The final results were resolved by discussion.

\section{Statistical Analysis}

Quantitative data in this study were analyzed with independent $t$-test for normally distributed variables and Mann-Whitney $U$-test for non-normally distributed variables. Results were presented as mean \pm standard deviation (SD) or median \pm range. Our quantitative data is presented with $\bar{x} \pm \mathrm{s}$. The independent sample $t$-test or corrected $t$-test (when the variance was uneven) was used for comparison between groups. The data that did not conform to the normal distribution was tested by the Mann-Whitney $U$-test, and the qualitative data was tested by the chi-square test or Fisher's exact test. No multiple comparison was applied, and the test standard was $P<0.05$ for statistical difference. Statistical analysis was performed by using SPSS 21.0 (SPSS Inc, Chicago, IL, USA).

\section{RESULTS}

\section{Clinical Characteristics}

Demographic and clinical features of 65 patients in our study were displayed in Table 1. There was no significant difference 
TABLE 1 | Clinical features of multiple sclerosis (MS) and primary angiitis of central nervous system (PACNS).

\begin{tabular}{lccc}
\hline & MS $(\boldsymbol{n}=\mathbf{4 9})$ & PACNS $(\boldsymbol{n}=\mathbf{1 6})$ & $\boldsymbol{p}$ \\
\hline Female percentage (\%) & $24(48.5 \%)$ & $8(50 \%)$ & 0.943 \\
Age at onset, years (SD) & $35.0 \pm 13.1$ & $32.0 \pm 18.3$ & 0.476 \\
$\quad$ Range (years) & $19-63$ & $20-70$ & \\
Disease duration (months) & $33.9 \pm 19.13$ & $13.7 \pm 9.39$ & 0.003 \\
$\quad$ Range & $6-84$ & $1-24$ & \\
Follow-up (months) & $25.3 \pm 9.8$ & $22.5 \pm 9.0$ & 0.501 \\
$\quad$ Range & $12-43$ & $14-40$ & \\
$\quad$ Relapses (SD) & $3 \pm 1.5$ & $2 \pm 1.2$ & - \\
Clinical features & & & \\
Headache (\%) & $5(11.4 \%)$ & $7(43.8 \%)$ & 0.003 \\
Visual impairment (\%) & $21(42.9 \%)$ & $2(12.5 \%)$ & 0.036 \\
Sensory symptoms (\%) & $15(30.6 \%)$ & $4(25.0 \%)$ & 0.761 \\
Limb weakness (\%) & $24(48.5 \%)$ & $4(25 \%)$ & 0.146 \\
\hline
\end{tabular}

TABLE 2 | Comparison of basal ganglia/thalamus lesions between multiple sclerosis (MS) and primary angiitis of central nervous system (PACNS).

\begin{tabular}{lccc}
\hline & MS & PACNS & $\boldsymbol{p}$ \\
\hline Cases involved, $\boldsymbol{n}$ (\%) & & & \\
Putamen & $19(38.8 \%)$ & $11(68.8 \%)$ & 0.037 \\
Caudatum & $8(16.3 \%)$ & $2(12.5 \%)$ & 1.000 \\
Globus pallidus & $9(18.4 \%)$ & $5(31.3 \%)$ & 0.276 \\
Claustrum & $3(6.1 \%)$ & $2(12.5 \%)$ & 0.590 \\
Thalamus & $41(83.7 \%)$ & $7(43.8 \%)$ & 0.002 \\
Internal capsule & $27(55.1 \%)$ & $6(37.5 \%)$ & 0.221 \\
External capsule & $8(16.3 \%)$ & $4(25.0 \%)$ & 0.470 \\
Diameter, mm (SD) & & & \\
Putamen & $9.3 \pm 5.4$ & $11.3 \pm 8.5$ & 0.437 \\
Caudatum & $9.6 \pm 4.3$ & $6.0 \pm 2.8$ & 0.299 \\
Globus pallidus & $6.4 \pm 3.9$ & $6.4 \pm 5.0$ & 0.986 \\
Thalamus & $7.9 \pm 3.7$ & $12.4 \pm 4.3$ & 0.006 \\
Internal capsule & $6.4 \pm 3.9$ & $11.9 \pm 6.1$ & 0.055 \\
External capsule & $13.6 \pm 6.0$ & $6.8 \pm 1.5$ & 0.053 \\
\hline
\end{tabular}

in onset age and gender distribution between MS and PACNS $(P>0.05)$. Patients with PACNS (43.8\%) presented with headache more often than MS $(11.4 \%)(P=0.003)$. Visual impairment was found in $21 \mathrm{MS}$ patients (42.9\%), but only in two PACNS sufferers $(12.5 \%)(P=0.036)$. Sensory disorder $(P$ $=0.761)$ and limb weakness $(P=0.146)$ were more prevalent in MS, but without differences, compared with PACNS.

\section{MRI Features}

As presented in Table 2, lesions showed more occurrence in PACNS cases, rather than in MS patients, in the regions of the putamen (68.8 vs. $38.8 \%)$ with significant difference $(P=0.037)$. For other areas, no differences were found between the two groups $(P>0.05)$.
We then examined the overall size of lesions and found that size of lesion in MS was much smaller than that in PACNS $(P=0.004)$. Considering the regions, MS had obviously smaller thalamus lesions than PACNS (MS vs. PACNS $7.9 \pm 3.7 \mathrm{~mm}$ vs. $12.4 \pm 4.3 \mathrm{~mm}, P=0.006)$. The diameter of the lesions located in putamen of MS was smaller than those from PACNS, but without any significance (MS vs. PACNS $9.3 \pm 5.4 \mathrm{~mm}$ vs. 11.3 $\pm 8.5 \mathrm{~mm}, P=0.437)$. There was also no significance between the two diseases, with respect to caudatum (MS vs. PACNS $9.6 \pm$ $4.3 \mathrm{~mm}$ vs. $6.0 \pm 2.8 \mathrm{~mm}, P=0.299$ ) and globus pallidus (MS vs. PACNS $6.4 \pm 3.9 \mathrm{~mm}$ vs. $6.4 \pm 5.0 \mathrm{~mm}, P=0.986)$. MS patients tended to have smaller lesions than PACNS patients in internal capsule (MS vs. PACNS $6.4 \pm 3.9 \mathrm{~mm}$ vs. $11.9 \pm 6.1 \mathrm{~mm}, P=$ 0.055), but with a low significance.

\section{DISCUSSION}

This study evaluated MRI characteristics of deep gray matter lesions in relapsing-remitting MS and PACNS. The results provided new radiological evidence for distinguishing MS from PACNS.

Although MS and PACNS are two different diseases considering their causes and etiologies, they can both express a chronic relapsing-remitting or progressive clinical course with MS-like symptoms, as well as brain abnormalities that are hard to distinguish from each other $(9,13)$. Moreover, PACNS remains challenging to diagnose, especially when small vessels are involved. So the value in this study is in helping to analyze the MRI characteristics in deep gray matter.

Deep gray matter is an important structure in the brain and is often affected in both Diseases (10, 14-16). Recently, the involvement of gray matter regions in MS demyelinating process has aroused much attention. In our study, the majority of DGML in basal ganglia had higher involvement in vasculitis than MS. Among them, the putamen was significantly more involved in primary CNS vasculitis. The insignificant variance in distribution of internal capsule lesions between the two groups might be a little perplexing at first. However, high involvement of internal capsule in MS and its correlation with disability, proven by Dalton et al. (17), made our results reasonable. Compared with PACNS patients, there is a higher number of thalamic lesions among patients with MS. However, this result should be interpreted with caution. Because we specifically selected MS patients with thalamic and/or basal ganglia lesions, not consecutive patients, the frequency of lesions' (e.g., thalamic lesions) appearance may not reflect the actual frequency of lesions' appearance. A further well-designed study with consecutive recruitment of patients is needed.

Thalamus and basal ganglia are also targeted by MS according to a number of studies $(18,19)$. Significant lymphocyte infiltration, complement deposition, and blood-brain barrier (BBB) disruption have so far not been detected in gray matter lesions, which makes it distinctive from the inflammatory process in white matter lesions (20). The less severe or even exemption of inflammation and BBB disruption in these regions makes the foci diameter in this location limited. This in a way offers an 
explanation for smaller lesions in the thalamus when compared with PACNS. Though the putamen was more frequently involved in vasculitis, the average diameter in MS was not distinctively smaller than in PACNS. The almost equal size of lesions in the internal and external gives us a hint that lesions from the basal ganglia might not be useful in making a differential diagnosis of MS and PACNS.

Also, there are several limitations to this study: (1) The number of PACNS patients in our study is small for the low morbidity of PACNS (2.4/million). Therefore, statistical deviation might happen; (2) There is inevitable bias caused by retrospective research; (3) No correction for multiple comparisons was performed in our study, so there is an increased risk for type 1 error; and (4) Selecting patients with thalamic and/or basal ganglia lesions does not allow for comparing the frequency of such lesions. This could be addressed in future studies by performing a consecutive recruitment of patients.

In conclusion, our study showed that involvement of the thalamus and putamen was useful in making differential diagnoses of MS and PACNS. MS tended to be affected in the thalamus, with smaller lesion sizes than PACNS. Since our study is an exploratory study, further studies are warranted to investigate the intrinsic mechanisms.

\section{REFERENCES}

1. Ravelli KG, Santos GD, Dos SN, Munhoz CD, Azzi-Nogueira D, Campos AC, et al. Nox2-dependent neuroinflammation in an EAE model of multiple sclerosis. Transl Neurosci. (2019) 10:1-9. doi: 10.1515/tnsci-2019-0001

2. Debernard L, Melzer TR, Alla S, Eagle J, Van Stockum S, Graham C, et al. Deep grey matter MRI abnormalities and cognitive function in relapsing-remitting multiple sclerosis. Psychiatry Res. (2015) 234:352-61. doi: 10.1016/j.pscychresns.2015.10.004

3. Mousavi MA, Ebrahim TF, Afghani A, Ashrafpour S, Dehghan S, Gol M, et al. Inhibition of GABA A receptor improved spatial memory impairment in the local model of demyelination in rat hippocampus. Behav Brain Res. (2018) 336:111-21. doi: 10.1016/j.bbr.2017.08.046

4. Shah MK, Mandayam S, Adrogue HJ. Osmotic demyelination unrelated to hyponatremia. Am J Kidney Dis. (2018) 71:436-40. doi: 10.1053/j.ajkd.2017.10.010

5. Eshaghi A, Prados F, Brownlee WJ, Altmann DR, Tur C, Cardoso MJ, et al. Deep gray matter volume loss drives disability worsening in multiple sclerosis. Ann Neurol. (2018) 83:210-22. doi: 10.1002/ana.25145

6. Damjanovic D, Valsasina P, Rocca MA, Stromillo ML, Gallo A, Enzinger $\mathrm{C}$, et al. Hippocampal and deep gray matter nuclei atrophy is relevant for explaining cognitive impairment in MS: a multicenter study. AJNR Am J Neuroradiol. (2017) 38:18-24. doi: 10.3174/ajnr.A4952

7. Marrodan M, Acosta JN, Alessandro L, Fernandez VC, Carnero CE, Arakaki $\mathrm{N}$, et al. Clinical and imaging features distinguishing Susac syndrome from primary angiitis of the central nervous system. J Neurol Sci. (2018) 395:29-34. doi: 10.1016/j.jns.2018.09.029

8. Scolding NJ, Wilson H, Hohlfeld R, Polman C, Leite I, Gilhus N. The recognition, diagnosis and management of cerebral vasculitis: a European survey. Eur J Neurol. (2002) 9:343-7. doi: 10.1046/j.1468-1331.2002.00422.x

9. Rolak LA, Fleming JO. The differential diagnosis of multiple sclerosis. Neurologist. (2007) 13:57-72. doi: 10.1097/01.nrl.0000254705.39956.34

10. Singhal AB, Topcuoglu MA, Fok JW, Kursun O, Nogueira RG, Frosch MP, et al. Reversible cerebral vasoconstriction syndromes and primary angiitis of

\section{DATA AVAILABILITY STATEMENT}

All datasets generated for this study are included in the article/supplementary material.

\section{ETHICS STATEMENT}

This study was approved by the local Ethics Committee of the Third Affiliated Hospital of Sun Yat-sen University (NO 2011-248). Informed consent for this investigation was obtained from the patients to evaluate the examination scores and standard laboratory test results that benefited the diagnosis and therapy.

\section{AUTHOR CONTRIBUTIONS}

$\mathrm{YC}$ and $\mathrm{AW}$ designed the study. $\mathrm{YC}, \mathrm{RL}$, and $\mathrm{ZH}$ performed the study. YC, WQ, and $\mathrm{XH}$ wrote the article. RL, AW, QY, and ZZ helped revise the manuscript. All authors contributed to the article and approved the submitted version.

\section{FUNDING}

This study was supported by grants from the National Natural Science Foundation of China (Grant No. 81701061).

the central nervous system: clinical, imaging, and angiographic comparison. Ann Neurol. (2016) 79:882-94. doi: 10.1002/ana.24652

11. Calabrese LH, Mallek JA. Primary angiitis of the central nervous system. Report of 8 new cases, review of the literature, and proposal for diagnostic criteria. Medicine (Baltimore). (1988) 67:20-39. doi: 10.1097/00005792-198801000-00002

12. Polman CH, Reingold SC, Edan G, Filippi M, Hartung HP, Kappos L, et al. Diagnostic criteria for multiple sclerosis: 2005 revisions to the "McDonald Criteria." Ann Neurol. (2005) 58:840-6. doi: 10.1002/ana. 20703

13. Maggi P, Absinta M, Grammatico M, Vuolo L, Emmi G, Carlucci G, et al. Central vein sign differentiates multiple sclerosis from central nervous system inflammatory vasculopathies. Ann Neurol. (2018) 83:283-94. doi: $10.1002 /$ ana.25146

14. Motl RW, Pilutti LA, Hubbard EA, Wetter NC, Sosnoff JJ, Sutton BP. Cardiorespiratory fitness and its association with thalamic, hippocampal, and basal ganglia volumes in multiple sclerosis. Neuroimage Clin. (2015) 7:661-6. doi: 10.1016/j.nicl.2015.02.017

15. Eijlers A, van Geest Q, Dekker I, Steenwijk MD, Meijer KA, Hulst HE, et al. Predicting cognitive decline in multiple sclerosis: a 5-year follow-up study. Brain. (2018) 141:2605-18. doi: 10.1093/brain/awy202

16. Tahedl M, Levine SM, Greenlee MW, Weissert R, Schwarzbach JV. Functional connectivity in multiple sclerosis: recent findings and future directions. Front Neurol. (2018) 9:828. doi: 10.3389/fneur.2018. 00828

17. Dalton CM, Chard DT, Davies GR, Miszkiel KA, Altmann DR, Fernando $\mathrm{K}$, et al. Early development of multiple sclerosis is associated with progressive grey matter atrophy in patients presenting with clinically isolated syndromes. Brain. (2014) 127:1101-7. doi: 10.1093/brain/ awh126

18. Doche E, Lecocq A, Maarouf A, Duhamel G, Soulier E, Confort-Gouny $S$, et al. Hypoperfusion of the thalamus is associated with disability in relapsing remitting multiple sclerosis. J Neuroradiol. (2017) 44:158-64. doi: 10.1016/j.neurad.2016.10.001 
19. Modica CM, Schweser F, Sudyn ML, Bertolino N, Preda M, Polak P, et al. Effect of teriflunomide on cortex-basal ganglia-thalamus (CxBGTh) circuit glutamatergic dysregulation in the Theiler's Murine Encephalomyelitis Virus mouse model of multiple sclerosis. PLoS ONE. (2017) 12:e182729. doi: 10.1371/journal.pone.0182729

20. Calabrese M, Magliozzi R, Ciccarelli O, Geurts JJ, Reynolds R, Martin R. Exploring the origins of grey matter damage in multiple sclerosis. Nat Rev Neurosci. (2015) 16:147-58. doi: 10.1038/nr n3900

Conflict of Interest: The authors declare that the research was conducted in the absence of any commercial or financial relationships that could be construed as a potential conflict of interest.
Publisher's Note: All claims expressed in this article are solely those of the authors and do not necessarily represent those of their affiliated organizations, or those of the publisher, the editors and the reviewers. Any product that may be evaluated in this article, or claim that may be made by its manufacturer, is not guaranteed or endorsed by the publisher.

Copyright $\odot 2021$ Chen, $L i, W u$, Qiu, Hu, Hu, Yang and Zhou. This is an open-access article distributed under the terms of the Creative Commons Attribution License (CC $B Y)$. The use, distribution or reproduction in other forums is permitted, provided the original author(s) and the copyright owner(s) are credited and that the original publication in this journal is cited, in accordance with accepted academic practice. No use, distribution or reproduction is permitted which does not comply with these terms. 